heterogeneous populations, lack of appropriate comparator treatments, and limited knowledge about the disease. Our review suggests that alternative research methods or tolerance of lower levels of evidence may be required.

\section{OP34 UNIVERSAL ANTENATAL CULTURE-BASED SCREENING FOR MATERNAL GROUP B STREPTOCOCCUS (GBS) CARRIAGE TO PREVENT EARLY-ONSET GBS DISEASE: A SYSTEMATIC REVIEW FOR THE UK NATIONAL SCREENING COMMITTEE (NSC)}

${ }^{1} \mathrm{~F}$ Seedat*, 'f Geppert, ${ }^{1} \mathrm{C}$ Stinton, ${ }^{1} \mathrm{~J}$ Patterson, ${ }^{2} \mathrm{CS}$ Brown, ${ }^{1} \mathrm{~B}$ Tan, ${ }^{1} \mathrm{~K}$ Freeman, ${ }^{1}$ OA Uthman, ${ }^{1}$ ND McCarthy, ${ }^{1}$ ER Robinson, ${ }^{1}$ SA Johnson, ${ }^{1} \mathrm{H}$ Fraser, ${ }^{1} \mathrm{~A}$ Clarke, ${ }^{1} \mathrm{SA}$ TaylorPhillips. 'Division of Health Sciences, The University of Warwick Medical School, Coventry, UK; ${ }^{2}$ Birmingham Public Health Laboratory, Heartlands Hospital, Birmingham, UK; ${ }^{3}$ Bacteria Reference Department, National Infection Service, Public Health England, London, UK

\subsection{6/jech-2017-SSMAbstracts.34}

Background GBS is the leading cause of morbidity and mortality from neonatal sepsis in the UK and patient groups are keen for screening to be implemented. Intrapartum antibiotic prophylaxis (IAP) is offered to women identified with GBS carriage or GBS risk factors to prevent mother to baby transmission and early-onset GBS disease (EOGBS, $<7$ days). This review on universal GBS screening for pregnant women was undertaken to assist NSC policy decision-making. Review questions were on: epidemiology of GBS, diagnostic accuracy of tests, effectiveness of IAP treatment, and effectiveness of universal GBS screening.

Methods Medline, Embase, and Cochrane databases were searched. Grey literature included Public Health England, British Paediatric Surveillance Unit, Audits and Confidential Enquiries, and reference lists of included papers. Participants were pregnant women $\geq 35$ weeks or neonates $<7$ days. The intervention was selective culture from recto-vaginal swabs at 35-37 weeks followed by IAP treatment for those who were culture positive. Reviewers independently screened records, extracted data, and assessed methodological quality using appropriate tools for each question, including QUADAS-2, Cochrane RoB, and RoBANS tools. Data were synthesised narratively.

Results 73 studies were included from 6287 references. EOGBS in the UK affects 0.57 per 1000 live births with a case fatality of $5.2 \%$. Twenty-two percent of EOGBS cases and $63 \%$ of deaths are in preterm births (many would be ineligible for screening). The natural history of GBS is not known. We estimate that universal GBS screening would be offered to approximately 718126 pregnant term women annually.Approximately $63347(57.7 \%)$ women who test positive in labour and $3282(8 \%)$ who test negative in labour would transmit GBS to their neonates, and approximately 350 $(0.5 \%)$ neonates would develop EOGBS. We estimate the positive predictive value of selective culture to detect EOGBS to be around $0.2 \%(350 / 150,806)$. More than 150450 (>99\%) women would be false positive and unnecessarily treated. Harms from IAP are unclear but will include antibiotic resistance and other possible health problems. There were no randomised controlled trials of the effectiveness of GBS screening and observational studies gave inconsistent results for EOGBS mortality and morbidity.
Conclusion EOGBS is an important health condition. However, tests are not accurate predictors of maternal GBS transmission, or of EOGBS. Evidence on the harms and benefits of GBS screening is limited. Universal screening is therefore not recommended.

\section{OP35 PRAGMATIC INTEGRATED TRIALS IN SCREENING: A BREAST SCREENING EXAMPLE INVOLVING 1.2 MILLION WOMEN}

${ }^{1} \mathrm{~S}$ Taylor-Phillips*, ${ }^{1} \mathrm{D}$ Jenkinson, ${ }^{1} \mathrm{~A}$ Clarke, ${ }^{2} \mathrm{M}$ Wallis. ${ }^{1}$ Warwick Medical School, University of Warwick, Coventry, UK; ${ }^{2}$ Cambridge Breast Unit, Cambridge Hospitals NHS Foundation Trust, Cambridge, UK

\subsection{6/jech-2017-SSMAbstracts.35}

Background Pragmatic integrated trials use routine data and systems to automate participant selection, randomisation, outcome measurements and/or other elements to deliver large trials at low cost. Here we present an example of a large pragmatic integrated trial in breast cancer screening, and discuss the situations in which these trials are appropriate and acceptable.

Methods The intervention was a simple change to the mammography test for breast screening. Interpreting whether screening mammogram show cancer is a difficult repetitive task that can result in missed cancers and false-positive recalls, and some studies have indicated that missed cancers may increase with time on task (the vigilance decrement). In the UK two readers independently evaluate each batch of mammograms to search for signs of cancer. The intervention was to change the order in which batches of mammograms were presented for interpretation, to reduce the effects of the vigilance decrement.

This was evaluated using a multicentre, double-blind, cluster randomised clinical trial at 46 breast screening centres in England for 1 year. Three hundred sixty readers participated. The primary outcome was cancer detection rate; secondary outcomes were rates of recall and disagreements between readers. Results 1194147 women who had screening mammograms were randomised (596 642 in the intervention group; 597 505 in the control group), and 10484 cases (0.88\%) of breast cancer were detected. There was no significant difference in cancer detection rate with 5272 cancers $(0.88 \%)$ detected in the intervention group vs 5212 cancers $(0.87 \%)$ detected in the control group (difference, 0.01\% points; $95 \% \mathrm{CI},-0.02 \%$ to $0.04 \%$ points). There was also no difference in recall rate, with 24681 [4.14\%] in intervention and $24894[4.17 \%]$ in the control group (difference, $-0.03 \%$ points; $95 \%$ CI, $-0.10 \%$ to $0.04 \%$ points). Patterns of cancer detection and recall with time since a break indicated that performance did not decline with time on task as predicted by the vigilance decrement theory. In fact, positive predictive value increased with time on task.

Discussion This pragmatic integrated trial in over 1 million women cost less than $£ 300 \mathrm{k}$, and demonstrates that in certain circumstances this study design is appropriate. Considerations when planning a pragmatic integrated trial include whether consent is required at the individual or institutional level, whether the relevant outcomes are available in routine data, and the cost of the intervention. 